\title{
Cigarette smoke exposure-associated alterations to non-coding RNA
}

\author{
Matthew A. Maccani ${ }^{1,2 *}$ and Valerie S. Knopik ${ }^{1,3}$ \\ ${ }^{1}$ Division of Behavioral Genetics, Rhode Island Hospital, Providence, RI, USA \\ ${ }^{2}$ Center for Alcohol and Addiction Studies, Brown University, Providence, RI, USA \\ ${ }^{3}$ Department of Psychiatry and Human Behavior, Brown University, Providence, RI, USA
}

\section{Edited by:}

Marissa A. Ehringer, University of

Colorado, USA

Reviewed by:

Flavia Pichiorri, The Ohio State

University, USA

Yanan Yang, M.D. Anderson Cancer

Center, USA

*Correspondence:

Matthew A. Maccani, Division of Behavioral Genetics, Rhode Island Hospital, Providence, RI 02903, USA. e-mail:matthew_maccani@brown.edu
Environmental exposures vary by timing, severity, and frequency and may have a number of deleterious effects throughout the life course. The period of in utero development, for example, is one of the most crucial stages of development during which adverse environmental exposures can both alter the growth and development of the fetus as well as lead to aberrant fetal programming, increasing disease risk. During fetal development and beyond, the plethora of exposures, including nutrients, drugs, stress, and trauma, influence health, development, and survival. Recent research in environmental epigenetics has investigated the roles of environmental exposures in influencing epigenetic modes of gene regulation during pregnancy and at various stages of life. Many relatively common environmental exposures, such as cigarette smoking, alcohol consumption, and drug use, may have consequences for the expression and function of non-coding RNA (ncRNA), important post-transcriptional regulators of gene expression. A number of ncRNA have been discovered, including microRNA (miRNA), Piwi-interacting RNA (piRNA), and long non-coding RNA (long ncRNA). The best-characterized species of ncRNA are miRNA, the mature forms of which are $\sim 22$ nucleotides in length and capable of post-transcriptionally regulating target mRNA utilizing mechanisms based largely on the degree of complementarity between miRNA and target mRNA. Because miRNA can still negatively regulate gene expression when imperfectly base-paired with a target mRNA, a single miRNA can have a large number of potential mRNA targets and can regulate many different biological processes critical for health and development. The following review analyzes the current literature detailing links between cigarette smoke exposure and aberrant expression and function of ncRNA, assesses how such alterations may have consequences throughout the life course, and proposes future directions for this intriguing field of research.

\section{GENE-ENVIRONMENT INTERACTIONS AND ENVIRONMENTAL EXPOSURES}

The interaction of genes and the environment, often hypothesized to be through epigenetic mechanisms, modulates an organism's reproductive fitness, response to external stimuli, and health. Throughout the life course, a number of exposures can influence an individual's development, health, and overall quality of life. Environmental exposures vary by timing, severity, and frequency and may have a number of deleterious effects throughout the life course. The period of in utero development, for example, is a crucial stage during which adverse environmental exposures can both alter the growth and development of the fetus as well as lead to aberrant fetal programming, increasing disease risk. During fetal development and beyond, the plethora of exposures, including nutrients, drugs, stress, and trauma, influence health, development, and survival. Recent research in environmental epigenetics has investigated the roles of environmental exposures in influencing epigenetic modes of gene regulation (Reamon-Buettner et al., 2008), and a special focus of this work has been on determining the effects of cigarette smoking on epigenetic mechanisms, such as non-coding RNA (ncRNA), and resulting downstream consequences as a result of this harmful exposure.

\section{CIGARETTE SMOKING}

One of the most common, potentially hazardous environmental exposures that negatively influences health and development is cigarette smoke exposure. The CDC has reported that almost $21 \%$ of adults in the United States smoked cigarettes in 2009, a number equaling $\sim 46$ million people (CDC, 2010). In contrast to the number of studies reporting a decrease in the overall prevalence of smoking in women in the United States in the past 20 years, other reports have found that the prevalence of smoking in young pregnant women has increased (Jaakkola et al., 2001; CDC, 2004; Mohsin and Bauman, 2005). Conservative estimates report that $12-15 \%$ of women smoke while pregnant (Cnattingius, 2004; Goodwin et al., 2007), and it is likely that far greater numbers are exposed to secondhand (or passive) cigarette smoke during various periods of their pregnancies. Exposure to maternal cigarette smoking while in utero is associated with an increased risk for respiratory disease (Cook and Strachan, 1999), an increased 
risk for cancer later in life (Doherty et al., 2009), and a number of deleterious neurobehavioral outcomes in infancy and beyond (Olds et al., 1994; Huijbregts et al., 2007; Knopik, 2009; KiechlKohlendorfer et al., 2010). When one combines these estimates of babies who experienced in utero exposure to cigarette smoke with the numbers of adults who smoke cigarettes, one realizes that the number of individuals exposed to cigarette smoke during at least one period of their lives is quite large and far greater than what might be expected at first consideration.

Previous work has found over 4,000 chemicals in a cigarette, including nicotine, benzo[a]pyrene, and carbon monoxide, and more than 40 of these chemicals have been established as known carcinogens (Thielen et al., 2008). Cigarette smoking has been linked to a number of diseases and disabling conditions, including heart disease and lung diseases (CDC, 2008). Furthermore, for every individual who dies from a disease associated with smoking, 20 more people battle at least one major illness attributable to smoking (CDC, 2003). Several studies have singled out tobacco use as the world's leading preventable cause of death (CDC, 2002, 2003, 2008). By some estimates, up to five million deaths worldwide can be attributed to smoking and current trend data predicts that tobacco use will lead to more than eight million deaths a year by 2030 (WHO, 2008). In the USA, tobacco use has been attributed to $20 \%$ of deaths per year which equates to $\sim 443,000$ deaths annually, and $\sim 49,000$ of these have been attributed to secondhand smoke exposure (CDC, 2008). On average, smokers die 13-14 years earlier than non-smokers (CDC, 2002), suggesting that cigarette smoking and cigarette smoke exposure leads to increased morbidity and mortality.

Recent research has broadened investigations of the effects of cigarette smoke exposure into determining the effects of not only primary (or "mainstream") cigarette smoke but also environmental (also called "secondhand," "passive," or "sidestream") cigarette smoke. Sidestream cigarette smoke comprises the major component of environmental cigarette smoke and is defined as smoke which goes into the air directly from a burning cigarette (as opposed to smoke which is directly inhaled ("mainstream" smoke) from the burning cigarette; NIOSH, 1991). A variety of studies have suggested that components of sidestream or environmental cigarette smoke (ECS) differ from those in mainstream smoke in both component type and concentration, with some investigators concluding that ECS may be as harmful or even more harmful than mainstream smoke (Schick and Glantz, 2005). Work is ongoing to investigate the potentially harmful effects of both mainstream and sidestream cigarette smoke.

Taken collectively, these data suggest that cigarette smoking and cigarette smoke exposure throughout the life course is one of the most common hazardous exposures, and more recently, work has suggested that epigenetic mechanisms may be especially responsive to environmental exposures such as cigarette smoke.

\section{EPIGENETICS: MODE BY WHICH ENVIRONMENTAL EXPOSURES INFLUENCE HEALTH OUTCOMES}

By definition, epigenetics is the field of research which studies changes in gene expression not caused by changes in the sequence of DNA (Bird, 2007). The emergence of a subfield of epigenetics, called "environmental epigenetics" (Reamon-Buettner et al.,
2008), focuses on studying modes of epigenetic regulation with the greater understanding that environmental exposures may affect such modes of epigenetic regulation as well. Throughout the life course, epigenetic mechanisms may be the mode by which environmental exposures influence the development, health, and survival of the individual. Research in both model systems and human cohorts has suggested that a variety of environmental exposures have consequences for gene expression through epigenetic modes of gene regulation. Particular focus has been placed on four modes of epigenetic regulation, namely DNA methylation, imprinting, histone modifications, and ncRNA-mediated gene regulation (Crane-Godreau et al., 2009). While the study of DNA methylation remains the most widely studied mode of epigenetic regulation, the study of ncRNA-mediated gene regulation has gained significant attention over the past two decades.

\section{NON-CODING RNA}

Since the earliest discoveries of RNA as a product of the transcription of DNA, many have hypothesized that RNA may not only act as the intermediate step on the pathway to protein but may also have a degree of regulatory activity itself. Of the species of ncRNA, the three best-characterized forms are microRNA (miRNA), Piwiinteracting RNA (piRNA), and long non-coding RNA (long ncRNA), with miRNA garnering especially great attention.

\section{microRNA}

In the early 1990s, two small regulatory RNAs, known as lin-4 and let-7 were shown to control the timing of larval development in C. elegans (Lee et al., 1993; Reinhart et al., 2000). These small ncRNAs, initially called "lin-4 and let-7 RNAs," have since been determined to be members of a class of endogenous RNAs found in a number of species, including worms, flies, and mammals and since have been renamed "microRNAs (miRNAs)" (Lagos-Quintana et al., 2001; Lau et al., 2001; Lee and Ambros, 2001). Additional studies suggested that these small ncRNAs capable of gene regulation are found in plants, mammals, green algae, and viruses (GriffithsJones et al., 2008). Depending on the species and particular form of ncRNA, names of these small ncRNA vary. miRNA are a distinct class of ncRNA and differ from other species of ncRNA in both how they are formed as well as their particular mechanism of action. miRNA are formed from precursor transcripts which fold back on themselves, forming hairpin structures.

As reviewed previously (Maccani and Marsit, 2009), miRNA are initially transcribed by RNA Polymerase II as part of longer transcripts termed primary miRNAs (pri-miRNAs) which include $5^{\prime}$ caps and $3^{\prime}$ poly(A) tails (Lee et al., 2002; Smalheiser, 2003; Cai et al., 2004). A hairpin is then formed from the miRNA portion of the pri-miRNA (Lee et al., 2002). The double-stranded RNA (dsRNA)-RNA-specific ribonuclease Drosha then digests the primiRNA and releases the hairpin which, post-Drosha processing, is referred to as a precursor-miRNA (pre-miRNA; Lee et al., 2003) and is $70-75$ nucleotides in length (Lee et al., 2003; Yi et al., 2003). pre-miRNA is subsequently exported from the nucleus to the cytoplasm by a complex containing Exportin-5 (Exp-5; Yi et al., 2003; Lund et al., 2004) and in the cytoplasm, is cleaved by Dicer (Lee et al., 2003; Yi et al., 2003) into a dsRNA with short 3' overhangs at the ends (Lund et al., 2004). After cleavage by Dicer, the 
Dicer-processed dsRNA must separate into two strands, and the single-stranded mature miRNA has to associate with the RNAinduced silencing complex (RISC). The RISC traffics the mature miRNA to its mRNA target where it effectively silences it (Hutvagner and Zamore, 2002). Additionally, work has revealed that determination of the active strand in the post-Dicer-processed dsRNA is directly related to the stability of dsRNA ends (Khvorova et al., 2003; Schwarz et al., 2003); more specifically, the strand with a less-stable base pairing of the two to four nucleotides at the $5^{\prime}$ end of the duplex tends to associate with the RISC and becomes the active miRNA which can be trafficked to its mRNA target to effectively post-transcriptionally silence that target (Schwarz et al., 2003). miRNA biosynthesis, as well as the post-transcriptional silencing of target mRNA, is summarized in Figure $\mathbf{1 .}$

Work has suggested that miRNA regulate gene expression posttranscriptionally by base pairing to a target mRNA. The specific mechanism of miRNA-mediated gene regulation depends on a variety of factors, the most important of which appears to be the degree of complementarity between the miRNA sequence and the target mRNA sequence (Lagos-Quintana et al., 2001)

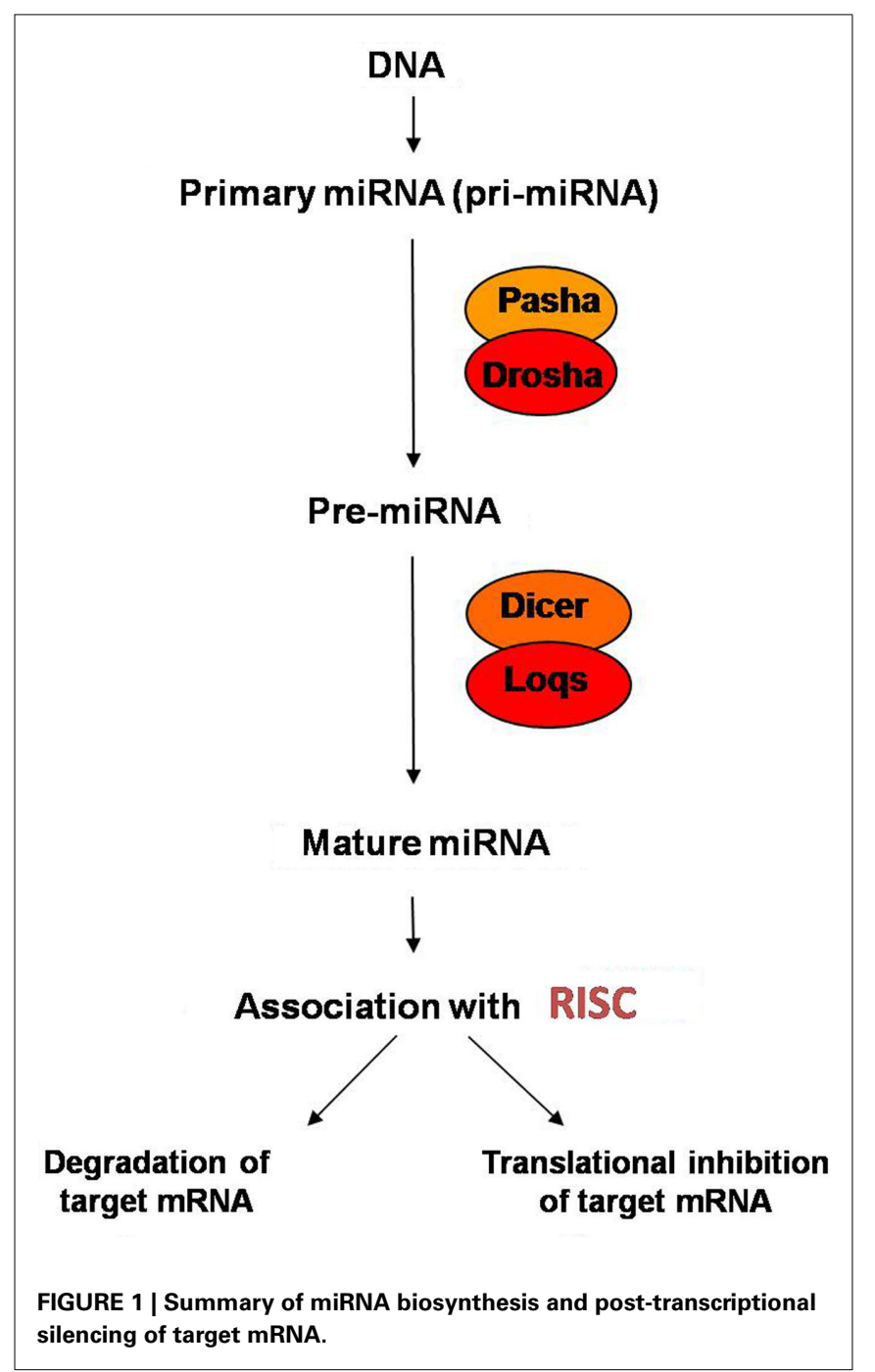

As described above, the mature miRNA's active strand associates with the Argonaute protein of the RISC and the RISC traffics the miRNA to its target mRNA (Hutvagner and Zamore, 2002; Mourelatos et al., 2002) where it post-transcriptionally regulates gene expression. Generally, a miRNA with perfect complementarity to the sequence of its target mRNA will lead to degradation of the mRNA transcript by a mechanism of Argonaute-catalyzed mRNA cleavage (Hutvagner and Zamore, 2002; Song et al., 2004; Yekta et al., 2004). A miRNA with imperfect sequence complementarity to its target mRNA will result in repression of translation of the target mRNA by blocking or altering the function of translational machinery (Lagos-Quintana et al., 2001) through mechanisms including the inhibition of translation initiation and poly(A) shortening (Filipowicz et al., 2008). Other observations have even suggested that miRNA can utilize a combination of both translational repression and mRNA degradation as the mechanism for their post-transcriptional gene regulation (Lim et al., 2005). Since partial or imperfect complementarity of a miRNA to a target mRNA can lead to translational repression which can effectively silence a gene, a single miRNA has the capability of regulating a large number of genes (Du and Zamore, 2007). By their mechanisms of negative regulation, miRNA have been shown to exhibit tissue-specific expression and function and play a role in regulating a wide range of biological processes, including differentiation, proliferation, apoptosis, and stress responses (Crane-Godreau et al., 2009).

\section{PIWI-INTERACTING RNA}

Another class of ncRNA that has recently been discovered is the class of piRNA. Compared to the miRNA and Argonaute protein complex, piRNAs associate with PIWI proteins to form RNAprotein complexes capable of gene regulation (Thomson and Lin, 2009). Several thousand piRNA, 24-32 nucleotides in length, have been discovered in zebrafish, Drosophila, and mammals. The majority of piRNA seem to be generated from a relatively small number of long single-stranded RNA precursors which are frequently encoded in repetitive intergenic sequences. PIWI proteins, and seemingly the piRNA which associate with them, are important for ensuring a number of key processes during germline development, including germline determination, spermiogenesis, and the silencing of transposons (Thomson and Lin, 2009). In mammals, piRNA have been detected in testes and ovaries but data suggest they may only be required for proper development in males (Aravin et al., 2006; Siomi et al., 2011).

In contrast with the relatively advanced understanding of the biogenesis of miRNA, the biogenesis of piRNA is not well understood. Recent work has suggested that in the case of pachytene piRNA, piRNA whose expression and function are important during the pachytene phase of meiosis, piRNA precursors are transcribed in a type of primary processing pathway (Brennecke et al., 2007; Seto et al., 2007; Aravin et al., 2008). Other work has suggested a type of "ping-pong" mechanism of biogenesis in which primary piRNA identify their mRNA targets and result in PIWI protein recruitment. This leads to the cleavage of the primary transcript at a location 10 nucleotides from the $5^{\prime}$ end of the primary piRNA, ultimately producing the secondary piRNA (Brennecke et al., 2007). Current data suggest that one or both of 
these proposed mechanisms explains the biogenesis of piRNA in a species-specific fashion with some degree of conservation across species (Faehnle and Joshua-Tor, 2007; Das et al., 2008; Klattenhoff and Theurkauf, 2008; Unhavaithaya et al., 2009).

In terms of biological importance, piRNA have been implicated in the silencing of transposons, and many piRNA are antisense to sequences of transposons (Malone and Hannon, 2009). In mammals, piRNA have been suggested to be most essential during embryonic development (Aravin et al., 2008), and in many species, data reveal that piRNA are required for spermatogenesis (Grimson et al., 2008). A more detailed characterization of both the biogenesis and mechanisms of action of piRNA will be valuable in further revealing how these small, but important, ncRNA function to regulate key periods of development.

\section{LONG nCRNA}

Long non-coding RNAs are longer than 200 nucleotides, and this characterization distinguishes long ncRNA from other forms of ncRNA, such as miRNA or piRNA. As has been described above, many forms of small ncRNA, such as miRNA or piRNA, show relatively strong conservation across species (Bentwich et al., 2005); long ncRNA, however, have been shown to lack such relatively strong conservation, leading to conclusions by early investigations that such long ncRNA were non-functional (Brosius, 2005; Struhl, 2007), theories which have been largely dismissed following subsequent analyses. Even two of the most well-characterized long ncRNA, Air and Xist, exhibit relatively poor conservation (Nesterova et al., 2001) but their roles are crucial to an organism's survival. As of late 2011, well over 100 long ncRNA have been characterized and annotated on lncRNAdb, a database which provides annotations of eukaryotic long ncRNA (Amaral et al., 2011).

One of the most well-characterized long ncRNAs is Xist (Wutz, 2007), and Xist has been shown to play a key role in X-chromosome inactivation in female placental mammals (Wutz, 2007). During the period of embryonic stem cell differentiation, Xist is expressed from the future inactive $\mathrm{X}$ chromosome and coats what then becomes the inactive $\mathrm{X}$ chromosome. Following expression of Xist, irreversible chromatin modifications including loss of histone (H3K9) acetylation and $\mathrm{H} 3 \mathrm{~K} 4$ methylation and establishment of repressive $\mathrm{H} 4$ hypoacetylation and $\mathrm{H} 3 \mathrm{~K} 27$ trimethylation which, along with other modifications, lead to the silencing of X-linked genes (Morey et al., 2004; Wutz, 2007). Collectively, the long ncRNA "Xist" and other ncRNAs expressed from Xist loci effectively allow only one $\mathrm{X}$ chromosome to be active in female placental mammals.

Research focused on understanding the roles of long ncRNA in health and disease is ongoing. Work to determine associations of dysregulated expression of long ncRNA with various tumors, as well as tumor differentiation, continues and will elucidate these important relationships (Pibouin et al., 2002; Reis et al., 2004; Fu et al., 2006; Lin et al., 2007). Specific roles of long ncRNA in driving or preventing tumorigenesis, however, have been largely undetermined. Future work focusing on enhanced understanding of how these long ncRNA are involved in regulating key cell processes throughout the life course, as well as how these long ncRNA are responsive to environmental exposures, such as cigarette smoke, will be key to better elucidating the function of long ncRNA.

\section{CIGARETTE SMOKE-ASSOCIATED EFFECTS ON ncRNA}

The influence of cigarette smoking and the effects of components of cigarette smoke on miRNA expression and function have been the most heavily studied aspects of research motivated to characterize the effects of cigarette smoke exposure on ncRNA. As of manuscript preparation, no studies have been published assessing associations of cigarette smoking or direct effects of components of cigarette smoke on piRNA, and relatively few studies have investigated the influence of smoke exposure on dysregulating long ncRNA expression. Since exposure to mainstream and sidestream cigarette smoke remains relatively common and potentially hazardous, investigations into effects on miRNA, piRNA, and long ncRNA may be crucial to elucidating currently poorly understood mechanisms. Such understanding may further explain how components of cigarette smoke may be mediating their effects through piRNA, long ncRNA, and other less-characterized species of ncRNA.

\section{MATERNAL CIGARETTE SMOKING DURING PREGNANCY AND ABERRANT EXPRESSION OF PLACENTAL MIRNA}

During fetal development, the placenta is of utmost importance to ensuring the proper growth and development of the fetus; it provides the fetus with nutrients, aids in the elimination of waste products, and protects the fetus from a variety of environmental toxicants (Crane-Godreau et al., 2009; Maccani et al., 2010). Recently, Maccani et al. (2010) used a candidate miRNA approach to investigate how maternal cigarette smoking during pregnancy might be associated with differential expression of miRNA in the placenta. Maccani and colleagues' data suggested that maternal cigarette smoking during pregnancy is associated with the downregulation of $m i R-16, m i R-21$, and $m i R-146 a$. In an attempt to further explore which components of cigarette smoke might modulate specific miRNA, Maccani and colleagues exposed three human placental cell lines from different stages of placental development to nicotine and benzo[a]pyrene, two components of cigarette smoke, and assessed candidate miRNA expression. The three human placental cell lines used were first trimester villous 3A cells, first trimester extravillous HTR8 cells, and the third trimester extravillous TCL- 1 cells; the three cell lines represent different periods and aspects of placental development and were selected to further investigate how different components of cigarette smoke might dysregulate miRNA expression in different placental cell types (Maccani et al., 2010). Data suggested that $m i R-146 a$ was downregulated in TCL-1 cells treated with nicotine and benzo[a]pyrene, suggesting that this particular miRNA may be particularly sensitive to agents of cellular stress and to these two components of cigarette smoke (Maccani et al., 2010; Maccani and Marsit, 2011).

As has been reviewed elsewhere (Maccani and Marsit, 2011), Maccani and colleagues' observations were limited by a relatively small set of samples $(n=25)$ and a lack of data regarding the duration of cigarette smoking during pregnancy or frequency of use, as well as more extensive environmental exposure information. Despite these limitations, Maccani and colleagues' findings comprise an important first step in investigating associations between maternal cigarette smoking during pregnancy and miRNA expression in the placenta. Future work is needed to 
investigate associations between maternal cigarette smoking during pregnancy and aberrant miRNA expression in a larger cohort of samples with more extensive exposure information (Maccani et al., 2010; Maccani and Marsit, 2011).

\section{CIGARETTE SMOKING AND ABERRANT EXPRESSION OF LUNG MIRNA}

Xi et al. (2010) investigated how cigarette smoke condensate (CSC) alters miRNA expression and function in normal human respiratory epithelial cells and lung cancer cells. For their experiments, $\mathrm{Xi}$ and colleagues prepared CSC from Kentucky Reference 3R4F research blend cigarettes (Liu et al., 2010) and resuspended the condensate at a concentration of $1 \mathrm{mg} \operatorname{tar} / \mathrm{mL}$ in RPMI. They found that exposure of cells to this CSC increased the expression of miR-31 in both normal respiratory epithelial cells and in lung cancer cells (Xi et al., 2010). Further results suggested that overexpression of miR-31 led to increased proliferation and tumorigenicity in lung cancer cells, whereas knockdown of miR-31 expression significantly inhibited growth of lung cancer cells (Xi et al., 2010). These findings suggest that miR-31 is both responsive to cigarette smoke exposure in normal respiratory epithelia and lung cancer cells and may play a role as an oncomir, or a miRNA whose expression and function may promote the development of cancer, in the pathway leading to carcinogenesis in the lung (Xi et al., 2010).

\section{CIGARETTE SMOKING AND DYSREGULATED mIRNA EXPRESSION IN HUMAN AIRWAY EPITHELIUM}

Schembri et al. (2009) investigated whole-genome miRNA expression in bronchial airway epithelium from current or never smokers $(n=20)$. They found that 28 miRNA were differentially expressed and a majority of these differentially expressed miRNA were downregulated in the airway epithelium of smokers. Furthermore, they investigated one candidate miRNA shown to be dysregulated due to smoke exposure, miR-218, by exposing primary bronchial epithelial cells to CSC, finding that such exposure downregulates miR-218 expression levels (Schembri et al., 2009). While Schembri and colleagues' sample set was limited in size, their work comprised an important first step in determining how miRNA may be a mechanism by which smoking dysregulates bronchial airway gene expression, potentially leading to downstream smoking-associated disease risk.

\section{ENVIRONMENTAL CIGARETTE SMOKE AND ABERRANT EXPRESSION OF miRNA}

While a number of studies have focused on the influences of primary cigarette smoking on miRNA expression and function, work is also being done to characterize the effects of ECS (also called "passive," "secondhand," or "sidestream" cigarette smoke) on miRNA expression. Three studies characterizing the effects of ECS on miRNA expression in the lung and liver were conducted by Izzotti et al. (2009, 2010a,b).

Izzotti et al. (2009) investigated the effects of ECS exposure on miRNA expression in the lungs of rats exposed to ECS for 28 days. They found that the most greatly downregulated miRNA belonged to miRNA families previously shown to regulate a number of key biological processes, including stress response, proliferation, angiogenesis, apoptosis, and others. Furthermore, they found that ECS exposure resulted in the upregulation of $2.9 \%$ of genes and $9.7 \%$ of proteins in the same tissue, suggesting that the ECS exposure-induced downregulation of miRNA may, in part, be leading to the increased protein levels of genes whose mRNA would have otherwise been targeted for post-transcriptional regulation.

In their two 2010 studies, Izzotti and colleagues exposed either neonatal mice (Izzotti et al., 2010b) or rats (Izzotti et al., 2010a) to ECS but also to chemopreventive agents and measured miRNA expression in the lung and the liver following exposure. Their data suggested that ECS dysregulated miRNA expression in lung and had a variety of mixed effects in liver. Phenethyl isothiocyanate and budesonide exposure was observed to protect the lung from ECSinduced dysregulated miRNA expression but had adverse effects in the liver (Izzotti et al., 2010b). Exposure to chemopreventive agents $\mathrm{N}$-acetylcysteine, oltipraz, indole-3-carbinol, 5-6-benzoflavone, and phenethyl isothiocyanate (as single exposures or in combinations) were shown to attenuate alterations in lung attributed to ECS (Izzotti et al., 2010a). Collectively, their findings further underscore the utility of miRNA profiles in various tissues as potential key tools for analyzing both protective and adverse effects of chemopreventive agents in mitigating the effects of exposure to ECS. Future work utilizing bioinformatically informed target prediction approaches may prove essential to better characterizing potential pathways dysregulated by aberrant miRNA expression and respective target $m R N A$ expression resulting from ECS. Future investigations into the molecular mechanisms by which ECS elicits downstream effects will be important for both understanding the effects of this potentially hazardous exposure as well as designing novel therapeutics to treat and improve outcome following exposure.

\section{Cigarette smoke exposure-associated dysregulation of long ncRNA}

Investigations into cigarette smoke exposure-associated dysregulation of miRNA have been relatively extensive compared to the few studies analyzing such alterations of long ncRNA. Silva et al. (2010) used whole-genome tiling arrays to investigate the upregulation of non-coding transcripts greater than 300 nucleotides in length in normal human bronchial epithelial cells (NHBE) exposed to the tobacco carcinogen nicotine-derived nitrosamine ketone (NNK), a component of tobacco smoke which can result in DNA damage. They found 12 long stress-induced non-coding transcripts (LSINCTs) which were upregulated in NHBE cells exposed to NNK. Follow-up analysis showed that several of these transcripts exhibit increased expression in many lung cancer and breast cancer cell lines. Analysis is ongoing to better characterize the functional aspects of these LSINCTs dysregulated by NNK. Other investigations have included using RNA-Seq technology to explore effects of smoking and lung cancer on the transcriptome of the airway (Beane et al., 2011) and characterizing the dysregulated monoallelic expression of the $\mathrm{H} 19$ gene in cigarette smokers' airway epithelium (Kaplan et al., 2003). These investigations all provide important first steps in characterizing the potential impacts of cigarette smoke exposure on long ncRNA; more extensive research will need to be conducted in the future to more comprehensively describe the effects of cigarette smoke exposure on long ncRNA in a variety of tissues. 


\section{CONCLUSION AND FUTURE DIRECTIONS}

Taken collectively, the body of literature describing our understanding of the effects of cigarette smoke on ncRNA expression and function is very much in its infancy. Studies to date have been relatively small in scope, have considered a relatively limited number of target tissues (i.e., placenta, lung, liver, and airway epithelial cells), and examined various types of cigarette smoke exposure (e.g., primary, passive/secondhand, and prenatal). While the variety in exposure types is informative and provides preliminary results, the relatively small sample sizes and limited number of studies overall make it clear that there is much more to be done in order to more definitively elucidate the effects of cigarette smoke (of any type) on ncRNA expression and the later downstream effects on behavioral health and other medical conditions.

The possible influence of epigenetic modification, including ncRNA expression, due to exposure to cigarette smoke of any kind (i.e., passive or secondhand, primary, or during pregnancy) on behavioral health and other medical conditions is manifold. As outlined above, the three best-characterized forms of ncRNA are microRNA (miRNA), piRNA, and long ncRNA, with miRNA garnering particularly great attention. In fact, at the time of the preparation of this review, there were virtually no published data on exposure-associated alterations to piRNA and relatively few studies on cigarette smoke-associated alterations to long ncRNA. Thus, unanswered questions include, but are not limited to, (i) the effects of cigarette smoke on piRNA and long ncRNA in multiple tissues, in animals and in humans, (ii) the effects of cigarette smoke on miRNA in the brain which might alter critical neurobehavioral circuitry in the developing brain or the adult brain, (iii) the influence of smoke exposure on ncRNA in asthma, lung cancer or other medical outcomes, and (iv) intergenerational transmission of smoking-related ncRNA changes (i.e., grandmaternal smoking influences which may affect germline cells and those epigenetic changes which may escape reprogramming during development). Use of animal models in mutually informative translational research (especially in inbred lines where genetic background is held constant) may further triangulate our ability as a field to investigate these issues (Knopik et al., Under Review).

It is clear, even from the relative paucity of research in this area, that ncRNA are biologically relevant and play an important role in the disease process. ncRNA alterations may also provide valuable information about therapeutic interventions. Yet, how do we align this information with the current state of science? One part of this answer lies in the research or clinical question of interest. If the question is whether cigarette smoke exposure, whether mainstream or sidestream, alters ncRNA expression and leads to the development of cancer cells, then the tissues of interest may indeed be relatively easily accessible. Animal models can be used to investigate ncRNA alterations in multiple tissues of interest, such as the lung, the esophagus, salivary glands, and bladder, but as with all model systems, consideration must be taken in study design regarding differential degrees of conservation of particular ncRNA across species. These preclinical models can be examined alongside human data, where biopsies of particular tissues may be available. However, if the outcome of interest is behavioral or psychiatric in nature (e.g., addiction, executive function, impulsive behavior, response to stress), the approach is less clear. Ideally, to consider the role of cigarette smoke exposure and ncRNA alterations on subsequent behavior, one would want to examine brain tissue. In humans, this is unavailable unless one considers post-mortem tissue; however, even then there is then the question of whether such investigations might be confounded due to potential ncRNA alterations associated with cause of death. There is considerable debate about the utility of blood as a biomarker for gene expression in brain and other tissues (Tsuang et al., 2005; Tian et al., 2009; Shivapurkar and Gazdar, 2010; Kukreja et al., 2011). In the search for a biomarker with clinical utility, blood does have certain advantages. Blood is an accessible tissue that can be relatively easily obtained, and while not a perfect representation of what might be expressed in brain, it can provide useful information for screening purposes. In either scenario, whether the health outcome is more psychological or somatic in nature, an additional question lies in what aspect of cigarette smoke leads to epigenetic alterations: more specifically, is it nicotine or one of the $4000+$ other xenobiotics (e.g., foreign substances) found in cigarette smoke - or one of the multitude of complex mixtures of these xenobiotics - which are most responsible for leading to epigenetic alterations? Carefully examining this piece of the equation will also be key to developing a better understanding of how components of cigarette smoke alter ncRNA expression and function, but also the utility of using such cigarette smoke-modulated ncRNA in diagnostic and therapeutic interventions.

In summary, increasing attention to the study of ncRNA and to "environmental epigenetics" (Reamon-Buettner et al., 2008) has inspired more researchers to embark on work to better understand how environmental exposures, such as cigarette smoke, affect ncRNA expression and function. Recommendations for future research include using both human cohorts and model systems to more comprehensively determine how the type, timing, frequency, duration, and degree of cigarette smoke exposure may alter miRNA, piRNA, and long ncRNA expression and function in a variety of tissues, thereby having the power to alter a number of health and developmental processes. The relative dearth of data demonstrating the effects of cigarette smoke on piRNA and relatively limited number of studies investigating the impact of cigarette smoke on long ncRNA underscore the need for future research to better describe such potentially hazardous effects of cigarette smoke on these two species of ncRNA and the processes they regulate. Hypothesis-generating approaches, such as microarray technology, when used in tandem with goldstandard validation approaches, such as Real-Time PCR, will be important for developing more agnostic study designs for discovering how ncRNA individually and collectively may be responsive to cigarette smoke exposure. Use of target prediction strategies combining in silico target prediction analysis with empirical target prediction confirmation (i.e., via Western blot) will enable researchers to better streamline their efforts to discover currently unknown targets of miRNA. Tools for predicting potential targets of piRNA and long ncRNA remain in early development and such bioinformatic tools will prove especially useful for further determining the functions of piRNA and long ncRNA, especially in the context of harmful environmental exposures. This 
will not only enhance understanding of how these harmful exposures impact health but may suggest the utility of ncRNA as both therapeutic targets and biomarkers for determining treatment efficacy. Together, these advances will be crucial for determining how alterations to the expression and function of ncRNA may be important modes by which environmental exposures, such as

\section{REFERENCES}

Amaral, P. P., Clark, M. B., Gascoigne, D. K., Dinger, M. E., and Mattick, J. S. (2011). lncRNAdb: a reference database for long noncoding RNAs. Nucleic Acids Res. 39, D146-D151.

Aravin, A., Gaidatzis, D., Pfeffer, S., Lagos-Quintana, M., Landgraf, P., Iovino, N., Morris, P., Brownstein, M. J., Kuramochi-Miyagawa, S., Nakano, T., Chien, M., Russo, J. J., Ju, J., Sheridan, R., Sander, C., Zavolan, M., and Tuschl, T. (2006). A novel class of small RNAs bind to MILI protein in mouse testes. Nature 442, 203-207.

Aravin, A. A., Sachidanandam, R., Bourc'his, D., Schaefer, C., Pezic, D., Toth, K. F., Bestor, T., and Hannon, G. J. (2008). A piRNA pathway primed by individual transposons is linked to de novo DNA methylation in mice. Mol. Cell 31, 785-799.

Beane, J., Vick, J., Schembri, F., Anderlind, C., Gower, A., Campbell, J., Luo, L., Zhang, X. H., Xiao, J., Alekseyev, Y. O., Wang, S., Levy, S., Massion, P. P., Lenburg, M., and Spira, A. (2011). Characterizing the impact of smoking and lung cancer on the airway transcriptome using RNA-Seq. Cancer Prev. Res. (Phila.) 4, 803-817.

Bentwich, I., Avniel, A., Karov, Y., Aharonov, R., Gilad, S., Barad, O., Barzilai, A., Einat, P., Einav, U., Meiri, E., Sharon, E., Spector, Y., and Bentwich, Z. (2005). Identification of hundreds of conserved and nonconserved human microRNAs. Nat. Genet. 37, 766-770.

Bird, A. (2007). Perceptions of epigenetics. Nature 447, 396-398.

Brennecke, J., Aravin, A. A., Stark, A., Dus, M., Kellis, M., Sachidanandam, R., and Hannon, G. J. (2007). Discrete small RNA-generating loci as master regulators of transposon activity in Drosophila. Cell 128, 1089-1103.

Brosius, J. (2005). Waste not, want not - transcript excess in multicellular eukaryotes. Trends Genet. 21, 287-288.

Cai, X., Hagedorn, C. H., and Cullen, B. R. (2004). Human microRNAs are processed from capped, polyadenylated transcripts that can also function as mRNAs. RNA 10, 1957-1966.

CDC. (2002). Annual smokingattributable mortality, years of potential life lost, and productivity losses - United States, 1995-1999. MMWR Morb. Mortal. Wkly. Rep. 51, 300-303.

CDC. (2003). Cigarette smokingattributable morbidity - United States, 2000. MMWR Morb. Mortal. Wkly. Rep. 52, 842-844.

CDC. (2004). Smoking during pregnancy - United States, 1990-2002. MMWR Morb. Mortal. Wkly. Rep. 53, 911-915.

CDC. (2008). Smoking-attributable mortality, years of potential life lost, and productivity losses - United States, 2000-2004. MMWR Morb. Mortal. Wkly. Rep. 57, 1226-1228.

CDC. (2010). Vital signs: current cigarette smoking among adults aged $\geq 18$ years-United States, 2009 . MMWR Morb. Mortal. Wkly. Rep. 1135-1140.

Cnattingius, S. (2004). The epidemiology of smoking during pregnancy: smoking prevalence, maternal characteristics, and pregnancy outcomes. Nicotine Tob. Res. 6(Suppl. 2), S125S140.

Cook, D. G., and Strachan, D. P. (1999). Health effects of passive smoking10: Summary of effects of parental smoking on the respiratory health of children and implications for research. Thorax 54, 357-366.

Crane-Godreau, M. A., Maccani, M. A., Eszterhas, S. K., Warner, S. L., Jukosky, J. A., and Fiering, S. (2009). Exposure to cigarette smoke disrupts CCL20-mediated antimicrobial activity in respiratory epithelial cells. Open. Immunol. J. 2, 86-93.

Das, P. P., Bagijn, M. P., Goldstein, L. D., Woolford, J. R., Lehrbach, N. J., Sapetschnig, A., Buhecha, H. R., Gilchrist, M. J., Howe, K. L., Stark, R., Matthews, N., Berezikov, E., Ketting, R. F., Tavaré, S., and Miska, E. A. (2008). Piwi and piRNAs act upstream of an endogenous siRNA pathway to suppress Tc3 transposon mobility in the Caenorhabditis elegans germline. Mol. Cell 31, 79-90.

Doherty, S. P., Grabowski, J., Hoffman, C., Ng, S. P., and Zelikoff, J. T. (2009). Early life insult from cigarette smoke may be predictive of chronic diseases later in life. Biomarkers 14(Suppl. 1), 97-101.

Du, T., and Zamore, P. D. (2007). Beginning to understand microRNA function. Cell Res. 17, 661-663.

cigarette smoke, influence health outcomes throughout the life course.

\section{ACKNOWLEDGMENTS}

This work was supported by R01DA023134 (Valerie S. Knopik) and 2 T32 AA 07459-26 (Matthew A. Maccani).

Faehnle, C. R., and Joshua-Tor, L. (2007). Argonautes confront new small RNAs. Curr. Opin. Chem. Biol. 11, 569-577.

Filipowicz, W., Bhattacharyya, S. N., and Sonenberg, N. (2008). Mechanisms of post-transcriptional regulation by microRNAs: are the answers in sight? Nat. Rev. Genet. 9, 102-114.

Fu, X., Ravindranath, L., Tran, N. Petrovics, G., and Srivastava, S. (2006). Regulation of apoptosis by a prostate-specific and prostate cancer-associated noncoding gene, PCGEM1. DNA Cell Biol. 25, 135-141.

Goodwin, R. D., Keyes, K., and Simuro, N. (2007). Mental disorders and nicotine dependence among pregnant women in the United States. Obstet. Gynecol. 109, 875-883.

Griffiths-Jones, S., Saini, H. K., van Dongen, S., and Enright, A. J. (2008). miRBase: tools for microRNA genomics. Nucleic Acids Res. 36, D154-D158.

Grimson, A., Srivastava, M., Fahey, B. Woodcroft, B. J., Chiang, H. R., King, N., Degnan, B. M., Rokhsar, D. S. and Bartel, D. P. (2008). Early origins and evolution of microRNAs and Piwi-interacting RNAs in animals. Nature 455, 1193-1197.

Huijbregts, S. C., Seguin, J. R., Zoccolillo, M., Boivin, M., and Tremblay, R. E. (2007). Associations of maternal prenatal smoking with early childhood physical aggression, hyperactivity-impulsivity, and their co-occurrence. J. Abnorm. Child. Psychol. 35, 203-215.

Hutvagner, G., and Zamore, P. D. (2002). A microRNA in a multipleturnover RNAi enzyme complex. Science 297, 2056-2060.

Izzotti, A., Calin, G. A., Arrigo, P., Steele, V. E., Croce, C. M., and De Flora, S. (2009). Downregulation of microRNA expression in the lungs of rats exposed to cigarette smoke. FASEB J. 23, 806-812.

Izzotti, A., Calin, G. A., Steele, V. E., Cartiglia, C., Longobardi, M., Croce, C. M., and De Flora, S. (2010a). Chemoprevention of cigarette smoke-induced alterations of MicroRNA expression in rat lungs. Cancer Prev. Res. (Phila.) 3, 62-72.

Izzotti, A., Larghero, P., Cartiglia, C., Longobardi, M., Pfeffer, U., Steele, V. E., and De Flora, S. (2010b).
Modulation of microRNA expression by budesonide, phenethyl isothiocyanate and cigarette smoke in mouse liver and lung. Carcinogenesis 31, 894-901.

Jaakkola, N., Jaakkola, M. S., Gissler, M. and Jaakkola, J. J. (2001). Smoking during pregnancy in Finland: determinants and trends, 1987-1997. Am. J. Public Health 91, 284-286.

Kaplan, R., Luettich, K., Heguy, A., Hackett, N. R., Harvey, B. G., and Crystal, R. G. (2003). Monoallelic up-regulation of the imprinted $\mathrm{H} 19$ gene in airway epithelium of phenotypically normal cigarette smokers. Cancer Res. 63, 1475-1482.

Khvorova, A., Reynolds, A., and Jayasena, S. D. (2003). Functional siRNAs and miRNAs exhibit strand bias. Cell 115, 209-216.

Kiechl-Kohlendorfer, U., Ralser, E., Pupp Peglow, U., Reiter, G., Griesmaier, E., and Trawoger, R. (2010). Smoking in pregnancy: a risk factor for adverse neurodevelopmental outcome in preterm infants? Acta Paediatr. 99, 1016-1019.

Klattenhoff, C., and Theurkauf, W. (2008). Biogenesis and germline functions of piRNAs. Development 135, 3-9.

Knopik, V. S. (2009). Maternal smoking during pregnancy and child outcomes: real or spurious effect? Dev. Neuropsychol. 34, 1-36.

Knopik, V. S., Maccani, M. A., and McGeary, J. E. (Under Review). The epigenetics of maternal cigarette smoking during pregnancy and effects on child development. Dev. Pscyhopathol.

Kukreja, R. C., Yin, C., and Salloum, F. N. (2011). MicroRNAs: new players in cardiac injury and protection. Mol. Pharmacol. 80, 558-564.

Lagos-Quintana, M., Rauhut, R., Lendeckel, W., and Tuschl, T. (2001). Identification of novel genes coding for small expressed RNAs. Science 294, 853-858.

Lau, N. C., Lim, L. P., Weinstein, E. G., and Bartel, D. P. (2001) An abundant class of tiny RNAs with probable regulatory roles in Caenorhabditis elegans. Science 294, 858-862.

Lee, R. C., and Ambros, V. (2001). An extensive class of small RNAs in Caenorhabditis elegans. Science 294, 862-864. 
Lee, R. C., Feinbaum, R. L., and Ambros, V. (1993). The C. elegans heterochronic gene lin-4 encodes small RNAs with antisense complementarity to lin-14. Cell 75 843-854.

Lee, Y., Ahn, C., Han, J., Choi, H., Kim, J., Yim, J., Lee, J., Provost, P., Rådmark, O., Kim, S., and Kim, V. N. (2003). The nuclear RNase III Drosha initiates microRNA processing. Nature 425, 415-419.

Lee, Y., Jeon, K., Lee, J. T., Kim, S., and Kim, V. N. (2002). MicroRNA maturation: stepwise processing and subcellular localization. EMBO J. 21, 4663-4670.

Lim, L. P., Lau, N. C., Garrett-Engele, P., Grimson, A., Schelter, J. M., Castle, J., Bartel, D. P., Linsley, P. S., and Johnson, J. M. (2005). Microarray analysis shows that some microRNAs downregulate large numbers of target mRNAs. Nature 433, 769-773.

Lin, R., Maeda, S., Liu, C., Karin, M., and Edgington, T. S. (2007). A large noncoding RNA is a marker for murine hepatocellular carcinomas and a spectrum of human carcinomas. Oncogene 26, 851-858.

Liu, F., Killian, J. K., Yang, M., Walker, R. L., Hong, J. A., Zhang, M., Davis, S., Zhang, Y., Hussain, M., Xi, S., Rao, M., Meltzer, P. A., and Schrump, D. S. (2010). Epigenomic alterations and gene expression profiles in respiratory epithelia exposed to cigarette smoke condensate. Oncogene 29, 3650-3664.

Lund, E., Guttinger, S., Calado, A., Dahlberg, J. E., and Kutay, U. (2004). Nuclear export of microRNA precursors. Science 303, 95-98.

Maccani, M. A., Avissar-Whiting, M., Banister, C. E., McGonnigal, B., Padbury, J. F., and Marsit, C. J. (2010). Maternal cigarette smoking during pregnancy is associated with downregulation of miR-16, miR-21, and miR-146a in the placenta. Epigenetics 5, 583-589.

Maccani, M. A., and Marsit, C. J. (2009). Epigenetics in the placenta. Am. J. Reprod. Immunol. 62, 78-89.

Maccani, M. A., and Marsit, C. J. (2011). Exposure and fetal growthassociated miRNA alterations in the human placenta. Clin. Epigenetics 2, 401-404.

Malone, C. D., and Hannon, G. J. (2009). Small RNAs as guardians of the genome. Cell 136, 656-668.

Mohsin, M., and Bauman, A. E. (2005). Socio-demographic factors associated with smoking and smoking cessation among 426,344 pregnant women in New South Wales, Australia. BMC Public Health 5, 138. doi:10.1186/1471-2458-5-138

Morey, C., Navarro, P., Debrand, E., Avner, P., Rougeulle, C., and Clerc, P.
(2004). The region $3^{\prime}$ to Xist mediates $\mathrm{X}$ chromosome counting and H3 Lys-4 dimethylation within the Xist gene. EMBO J. 23, 594-604.

Mourelatos, Z., Dostie, J., Paushkin, S., Sharma, A., Charroux, B., Abel, L., Rappsilber, J., Mann, M., and Dreyfuss, G. (2002). miRNPs: a novel class of ribonucleoproteins containing numerous microRNAs. Genes Dev. 16, 720-728.

Nesterova, T. B., Barton, S. C., Surani, M. A., and Brockdorff, N. (2001). Loss of Xist imprinting in diploid parthenogenetic preimplantation embryos. Dev. Biol. 235, 343-350.

NIOSH. (1991). Environmental Tobacco Smoke in the Workplace. Current Intelligence Bulletin 54. National Institute for Occupational Safety and Health (NIOSH), Cincinnati, 91-108.

Olds, D. L., Henderson, C. R. Jr., and Tatelbaum, R. (1994). Intellectual impairment in children of women who smoke cigarettes during pregnancy. Pediatrics 93, 221-227.

Pibouin, L., Villaudy, J., Ferbus, D., Muleris, M., Prosperi, M. T., Remvikos, Y., and Goubin, G. (2002). Cloning of the mRNA of overexpression in colon carcinoma1: a sequence overexpressed in a subset of colon carcinomas. Cancer Genet. Cytogenet. 133, 55-60.

Reamon-Buettner, S. M., Mutschler, V., and Borlak, J. (2008). The next innovation cycle in toxicogenomics: environmental epigenetics. Mutat. Res. 659, 158-165.

Reinhart, B. J., Slack, F. J., Basson, M., Pasquinelli, A. E., Bettinger, J. C., Rougvie, A. E., Horvitz, H. R., and Ruvkun, G. (2000). The 21-nucleotide let-7 RNA regulates developmental timing in Caenorhabditis elegans. Nature 403, 901-906.

Reis, E. M., Nakaya, H. I., Louro, R., Canavez, F. C., Flatschart, A. V., Almeida, G. T., Egidio, C. M., Paquola, A. C., Machado, A. A., Festa, F., Yamamoto, D., Alvarenga, R., da Silva, C. C., Brito, G. C., Simon, S. D., Moreira-Filho, C. A., Leite, K. R., Camara-Lopes, L. H., Campos, F. S., Gimba, E., Vignal, G. M., ElDorry, H., Sogayar, M. C., Barcinski, M. A., da Silva, A. M., and Verjovski-Almeida, S. (2004). Antisense intronic non-coding RNA levels correlate to the degree of tumor differentiation in prostate cancer. Oncogene 23, 6684-6692.

Schembri, F., Sridhar, S., Perdomo, C., Gustafson, A. M., Zhang, X., Ergun, A., Lu, J. N., Liu, G., Zhang, X. H., Bowers, J., Vaziri, C., Ott, K., Sensinger, K., Collins, J. J., Brody,
J. S., Getts, R., Lenburg, M. E. and Spira, A. (2009). MicroRNAs as modulators of smoking-induced gene expression changes in human airway epithelium. Proc. Natl. Acad. Sci. U.S.A. 106, 2319-2324.

Schick, S., and Glantz, S. (2005). Philip Morris toxicological experiments with fresh sidestream smoke: more toxic than mainstream smoke. Tob. Control 14, 396-404.

Schwarz, D. S., Hutvagner, G., Du, T., $\mathrm{Xu}, \mathrm{Z}$., Aronin, N., and Zamore, P. D. (2003). Asymmetry in the assembly of the RNAi enzyme complex. Cell 115, 199-208

Seto, A. G., Kingston, R. E., and Lau, N. C. (2007). The coming of age for Piwi proteins. Mol. Cell 26, 603-609.

Shivapurkar, N., and Gazdar, A. F. (2010). DNA methylation based biomarkers in non-invasive cancer screening. Curr. Mol. Med. 10, 123-132.

Silva, J. M., Perez, D. S., Pritchett, J. R., Halling, M. L., Tang, H., and Smith, D. I. (2010). Identification of long stress-induced noncoding transcripts that have altered expression in cancer. Genomics 95, 355-362.

Siomi, M. C., Sato, K., Pezic, D., and Aravin, A. A. (2011). PIWIinteracting small RNAs: the vanguard of genome defence. Nat. Rev. Mol. Cell Biol. 12, 246-258.

Smalheiser, N. R. (2003). EST analyses predict the existence of a population of chimeric microRNA precursormRNA transcripts expressed in normal human and mouse tissues. Genome Biol. 4, 403.

Song, J. J., Smith, S. K., Hannon, G. J., and Joshua-Tor, L. (2004). Crystal structure of argonaute and its implications for RISC slicer activity. Science 305, 1434-1437.

Struhl, K. (2007). Transcriptional noise and the fidelity of initiation by RNA polymerase II. Nat. Struct. Mol. Biol. 14, 103-105.

Thielen, A., Klus, H., and Muller, L. (2008). Tobacco smoke: unraveling a controversial subject. Exp. Toxicol. Pathol. 60, 141-156.

Thomson, T., and Lin, H. (2009). The biogenesis and function of PIWI proteins and piRNAs: progress and prospect. Annu. Rev. Cell Dev. Biol. 25, 355-376.

Tian, Z., Palmer, N., Schmid, P., Yao H., Galdzicki, M., Berger, B., Wu, E., and Kohane, I. S. (2009). A practical platform for blood biomarker study by using global gene expression profiling of peripheral whole blood. PLoS ONE 4, e5157. doi:10.1371/journal.pone.0005157

Tsuang, M. T., Nossova, N., Yager, T., Tsuang, M. M., Guo, S. C., Shyu, K.
G., Glatt, S. J., and Liew, C. C. (2005). Assessing the validity of blood-based gene expression profiles for the classification of schizophrenia and bipolar disorder: a preliminary report. Am. J. Med. Genet. B Neuropsychiatr. Genet. 133B, 1-5.

Unhavaithaya, Y., Hao, Y., Beyret, E., Yin, H., Kuramochi-Miyagawa, S., Nakano, T., and Lin, H. (2009). MILI, a PIWI-interacting RNAbinding protein, is required for germ line stem cell self-renewal and appears to positively regulate translation. J. Biol. Chem. 284, 6507-6519.

WHO. (2008). WHO Report on the Global Tobacco Epidemic, 2009. Geneva: World Health Organization. Wutz, A. (2007). Xist function: bridging chromatin and stem cells. Trends Genet. 23, 457-464.

Xi, S., Yang, M., Tao, Y., Xu, H., Shan, J., Inchauste, S., Zhang, M., Mercedes, L., Hong, J. A., Rao, M., and Schrump, D. S. (2010). Cigarette smoke induces C/EBP-betamediated activation of miR-31 in normal human respiratory epithelia and lung cancer cells. PLoS ONE 5, e13764. doi:10.1371/journal.pone.0013764

Yekta, S., Shih, I. H., and Bartel, D. P. (2004). MicroRNA-directed cleavage of HOXB8 mRNA. Science 304, 594-596.

Yi, R., Qin, Y., Macara, I. G., and Cullen, B. R. (2003). Exportin-5 mediates the nuclear export of premicroRNAs and short hairpin RNAs. Genes Dev. 17, 3011-3016.

Conflict of Interest Statement: The authors declare that the research was conducted in the absence of any commercial or financial relationships that could be construed as a potential conflict of interest.

Received: 03 February 2012; paper pending published: 21 February 2012, accepted: 20 March 2012; published online: 09April 2012.

Citation: Maccani MA and Knopik VS (2012) Cigarette smoke exposureassociated alterations to non-coding RNA. Front. Gene. 3:53. doi: 10.3389/fgene.2012.00053

This article was submitted to Frontiers in Non-Coding RNA, a specialty of Frontiers in Genetics.

Copyright (C) 2012 Maccani and Knopik. This is an open-access article distributed under the terms of the Creative Commons Attribution Non Commercial License, which permits non-commercial use, distribution, and reproduction in other forums, provided the original authors and source are credited. 